\title{
A peculiar bifurcation transition route of thermocapillary convection in rectangular liquid layers
}

\author{
Huan Jiang, Li Duan*, Qi Kang* \\ Key Laboratory of Microgravity, Institute of Mechanics, Chinese Academy of Sciences, Beijing 100190, PR China \\ School of Engineering Sciences, University of Chinese Academy of Sciences, Beijing 100049, PR China
}

\section{A R T I C L E I N F O}

\section{Article history:}

Received 27 July 2016

Received in revised form 7 March 2017

Accepted 7 March 2017

Available online 14 March 2017

\section{Keywords:}

Thermocapillary convection

Temperature oscillation

Transition route

Tangent bifurcation

Chaotic characteristic

\begin{abstract}
A B S T R A C T
Transition routes of thermocapillary convection are complex and diversiform in rectangular layers. In general, three types of transition routes were observed in thermocapillary convection, including quasiperiodic bifurcations, period-doubling bifurcations and tangent bifurcations. The tangent bifurcation is a peculiar type of bifurcations, which often appears accompanied with other types of bifurcation sequences. In our experiments, a detailed research on tangent bifurcations of thermocapillary convection in rectangular liquid layers was conducted through the measurement of single-point temperature oscillation in liquid. There are different tangent bifurcation series for various of experimental conditions, including different Prandtl numbers of silicone oil and different aspect ratios. It has certain occasionality for appearance of the tangent bifurcation, mainly in the experimental condition of Prandtl number of silicone oil equal to 16 ( $1 \mathrm{cSt}$ ) or Prandtl number equal to 25 (1.5 cSt). Chaotic characteristics of bifurcations are investigated by the method of phase-space reconstruction, maximum Lyapunov exponent and permutation entropy.
\end{abstract}

(c) 2017 Published by Elsevier Inc.

\section{Introduction}

The thermocapillary convection is driven by non-uniformity of surface tension, which comes from non-uniformity of surface temperature [1]. Thermocapillary-buoyancy flow is the main form of natural convection. Transition between the steady and the oscillatory states involves a nonlinear instability process [2]. A bifurcation is a complex structure in the nonlinear system. Deep investigations of this nonlinear phenomenon are of great benefit to understand the nonlinear behavior. In the past few decades, this classic physical phenomenon has attracted many researchers' interest. In general, there are three types of bifurcations in chaotic dynamics: period-doubling bifurcations, quasi-periodic bifurcations and tangent bifurcations. These bifurcations have been found in various convection including Rayleigh-Bénard convection and thermocapillary convection. Gollub and Benson [3] have done the experiment on Rayleigh-Bénard convection and research transition routes from laminar to turbulent flow. They observed four routes to non-periodic motion, including quasi-periodic motion at two frequencies-phase locking or entrainment, period doubling bifurcations of a periodic flow, quasi-periodic motion of three generally

\footnotetext{
* Corresponding authors at: Key Laboratory of Microgravity, Institute of Mechanics, Chinese Academy of Sciences, Beijing 100190, PR China.

E-mail addresses: duanli@imech.ac.cn (L. Duan), kq@imech.ac.cn (Q. Kang).
}

incommensurate frequencies, and the process of intermittent nonperiodicity by varying the geometrical aspect ratio, Prandtl number, and mean flow. Mukutmoni [4] has reported the numerical study on bifurcation sequences in Rayleigh-Bénard convection. He investigated the counter-intuitive transition route: steady state - periodic - quasi-periodic - steady state. Bucchignani and Fulvio $[5,6]$ has detected three different bifurcation sequences, but only identified two individual mechanisms for the transition to the non-periodic motion: the subharmonic cascade and the quasiperiodicity with three incommensurate frequencies. Li et al. [7] reported the numerical results about the transition to chaos in double-diffusive Marangoni convection in a rectangular cavity with horizontal temperature and concentration gradients. They found that the supercritical solution branch takes a quasiperiodicity and phase locking route to chaos while the subcritical branch follows the Ruelle-Takens-Newhouse scenario. Transient intermittency in the supercritical branch is observed and physical instability mechanisms of the subcritical branch are identified. Yu et al. [8] reported a counter-intuitive transition route from a perspective of the flow field in the capillary flow. They considered that the reverse transition from the three-dimensional unsteady flow to the steady flow is the reason that the spatial complexity of the flow increases as the thermal Marangoni number increases. Hu et al. [9] have researched the liquid-bridge model of the floating half zone, and reported the experimental and numerical results of 


\begin{tabular}{|c|c|c|}
\hline \multicolumn{3}{|c|}{ Nomenclature } \\
\hline$d$ & thickness of the liquid layer, mm & the embedding dimension \\
\hline$t$ & time, s & the permutation entropy \\
\hline$L$ & the length of the liquid layer between the cold-side and & \\
\hline & hot-side, mm & Greek symbols \\
\hline$g$ & gravitational acceleration, $\mathrm{m} \cdot \mathrm{s}^{-2}$ & $\lambda_{i} \quad$ the Lyapunov exponent \\
\hline$T$ & temperature of the measurement point, ${ }^{\circ} \mathrm{C}$ & kinematic viscosity, $\mathrm{m}^{2} \cdot \mathrm{s}^{-1}$ \\
\hline$\Delta T$ & $\begin{array}{l}\text { applied temperature difference between the two side- } \\
\text { walls }{ }^{\circ} \mathrm{C}\end{array}$ & thermal expansion coefficient, ${ }^{\circ} \mathrm{C}^{-1}$ \\
\hline $\operatorname{Pr}$ & Prandtl number of silicone oil & $\begin{array}{l}\text { thermal diffusivity, } \mathrm{m}^{2} \cdot \mathrm{s}^{-1} \\
\text { density } \mathrm{kg} \cdot \mathrm{m}^{-3}\end{array}$ \\
\hline$M a$ & Marangoni number of thermocapillary convection & surface tension, $\mathrm{N} \cdot \mathrm{m}^{-1}$ \\
\hline$f_{1}$ & the first fundamental frequency, $\mathrm{Hz}$ & aspect ratios of the liquid layer \\
\hline & the second fundamental frequency, $\mathrm{Hz}$ & the time-delay of the phase-space reconstruction \\
\hline
\end{tabular}

the transition process and the period-doubling bifurcation transition route in oscillatory thermocapillary convections. Zhu et al. [10] did some research on thermocapillary convection in rectangular liquid layers, and observed two types of transition routes, the period-doubling bifurcation and the quasi-periodic bifurcation. In addition, Zhu et al. [11] also researched characteristics of surface oscillation in the same experimental model.

The research about transition routes of thermocapillary convection has been a difficult job all the time, especially in rectangular liquid layers. Due to the weakness of temperature oscillations and the complexity and diversity of transition processes, our study has been immensely challenging. The aim of the present research work is to explore and summarize different types of bifurcations in transition routes of thermocapillary convection on the basis of our previous research work [10].

In the present paper, an important experimental finding is the tangent bifurcation transition route. Different types of tangent bifurcation transition cascades could be observed obviously in our experiments, and chaotic characteristics of tangent bifurcations could be analyzed in the phase-space.

\section{Experimental setup and techniques}

In order to research on evolvement of temperature oscillation in the experiment, we constructed a thermocapillary convection system as shown in Fig. 1.

It consists of a rectangular container lateral heated, a temperature controller system and a temperature measurement system. The rectangular container is made of $\mathrm{K} 9$ optical glass with the thickness of $6 \mathrm{~mm}$. The size of the rectangular container is $52 \mathrm{~mm} \times 36 \mathrm{~mm} \times 6 \mathrm{~mm}$, and the left cold end is a copper wall cooled by a semiconductor radiator while the right end is the same copper wall heated by an electrothermal film, as is shown in Fig. 2. The two ends of the rectangular container are controlled through two T-type thermocouples connected with Eurotherm 904P temperature controllers, which is the temperature controller system. The temperature measurement system consists of T-type thermocouples, the Keithley 2182A Nanovoltmeter and a LabVIEW software platform. The filament diameter of T-type thermocouples is $60 \mu \mathrm{m}$, and the size of welding head is $0.2 \mathrm{~mm}$. The thermocouple as a sensor transfers the temperature signal to voltage signal which could be measured by the Nanovoltmeter. Then the temperature oscillation signal could be imported into the LabVIEW software platform shown in Fig. 3 that could calculate power spectra of the temperature signal with the fast Fourier transform and timefrequency spectra of temperature oscillation series with the short-time Fourier transform. The sensitivity of the temperature measurement system is $0.001{ }^{\circ} \mathrm{C}$.In our experiment, the sampling frequency is $5 \mathrm{~Hz}$ due to the fundamental frequency of temperature oscillation is rather small.

For a single-point measurement mode, the selection of measuring point position is of vital importance. In the experiment, the measuring point is located in the middle of liquid layers and kept away from the side wall, which also could avoid the influence of boundary layers. In addition, a Plexiglas lid is put on the rectangular container, which can reduce the influence of evaporation of fluid in convection. Therefore, a steady thickness of the liquid layer can be maintained in the experimental run. There is a slit in the middle of the Plexiglas lid, which can be passed through by the thermocouple across a plastic tubing fixed in a holder. The holder with a spiral micrometer can rise and fall through regulating the scale, and then thermocouples could be located in the appropriate position according to our experimental needs. In addition, we have different arrangements for thermocouples in various location of

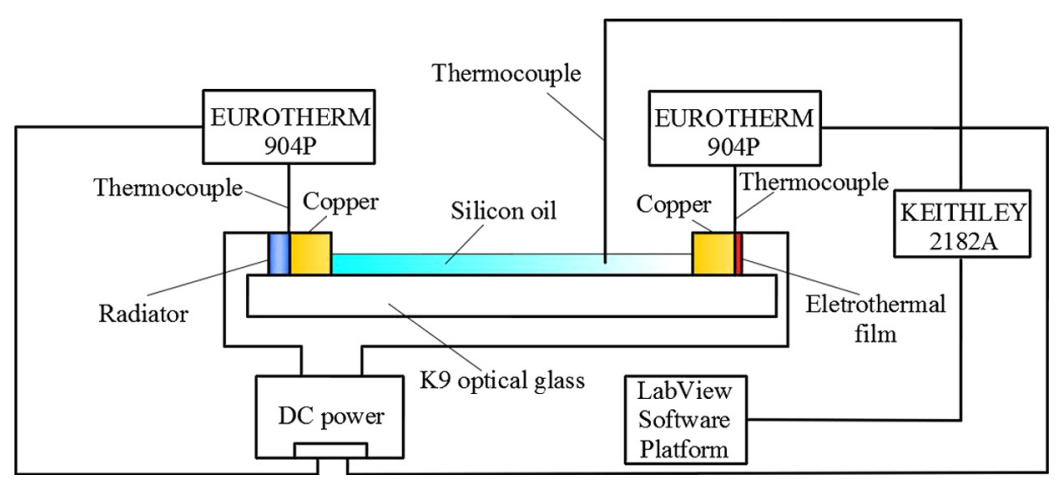

Fig. 1. The controlling system of thermocapillary convection. 


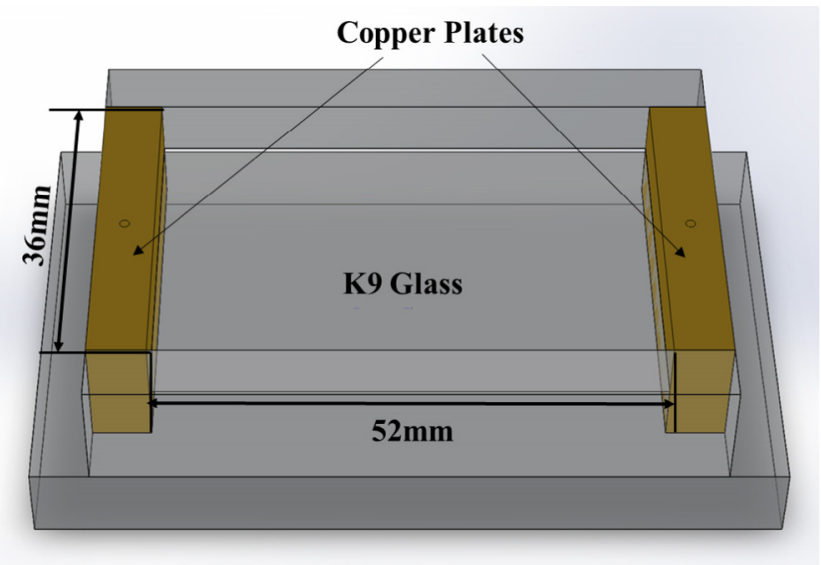

Fig. 2. The picture of a rectangular liquid container.

liquid layers in the experiment. The sound experimental results show that the same flow characteristic has been measured on the basis of the collected data, which indicates that thermocouples do not affect the thermocapillary convection.

To conduct the measurement on temperature oscillation steadily, the step-heating mode has been adopted over the experimental run and the step interval of temperature difference is $0.5^{\circ} \mathrm{C}$. Gradually escalate temperature difference between the hot wall and the cold wall to establish the convection system. When the target temperature difference has been attained, a period of constant temperature difference is maintained. Then the power spectrum analysis on temperature oscillation in each stage of constant temperature differences has been carried out, which could avoid and exclude the transient effect of the step-heating mode in the experiment.
The working fluid used in our experiments is Shin-Etsu KF96 silicone oil of $1 \mathrm{cSt}$ and $1.5 \mathrm{cSt}$, which could be characterized by Prandtl number (Pr). Physical properties of silicone oil are listed in Table 1. In addition, we could control over the influence of buoyancy by changing the thickness of the layer, which is defined as $\Gamma=L / d$. Here $L$ is the length of the liquid layer between the coldside and hot-side, and $d$ is the thickness of the liquid layer. The strength of thermocapillary convection is characterized by the Marangoni number, $M a=\frac{(\partial \sigma / \partial T)(\Delta T / L) d^{2}}{\rho v \kappa}$, where $\rho$ is the density, $v$ is the kinematic viscosity, $\kappa$ is the thermal diffusivity, $\partial \sigma / \partial T$ is the temperature coefficient of surface tension, and $\Delta T$ is the temperature difference between the cold-side and hot-side.

\section{Results and discussion}

Many transition routes in our experiments could be observed, as with the experimental findings of Gollub and Benson [3]. Tangent bifurcation is a peculiar form of bifurcations in our experiments, which rarely appears in previous experiments. In principle, tangent bifurcations will appear accompanied with other types of bifurcations, and it may be correlated with various types of intermittency [12].

\subsection{Transition routes}

The first transition route is the tangent bifurcation sequence only. When $\operatorname{Pr}=25$ and $\Gamma=17.3$, thermocapillary convection of a rectangular liquid layer follows the tangent bifurcation transition route. The evolution process could be observed distinctly from time-frequency spectrum in Fig. 4. Through time-frequency spectrum of the temperature oscillation series, the transition process of thermocapillary convection could be divided into different phases: the periodic bifurcation, the period-tripling bifurcation, and the period-sextupling bifurcation. At the beginning, there is

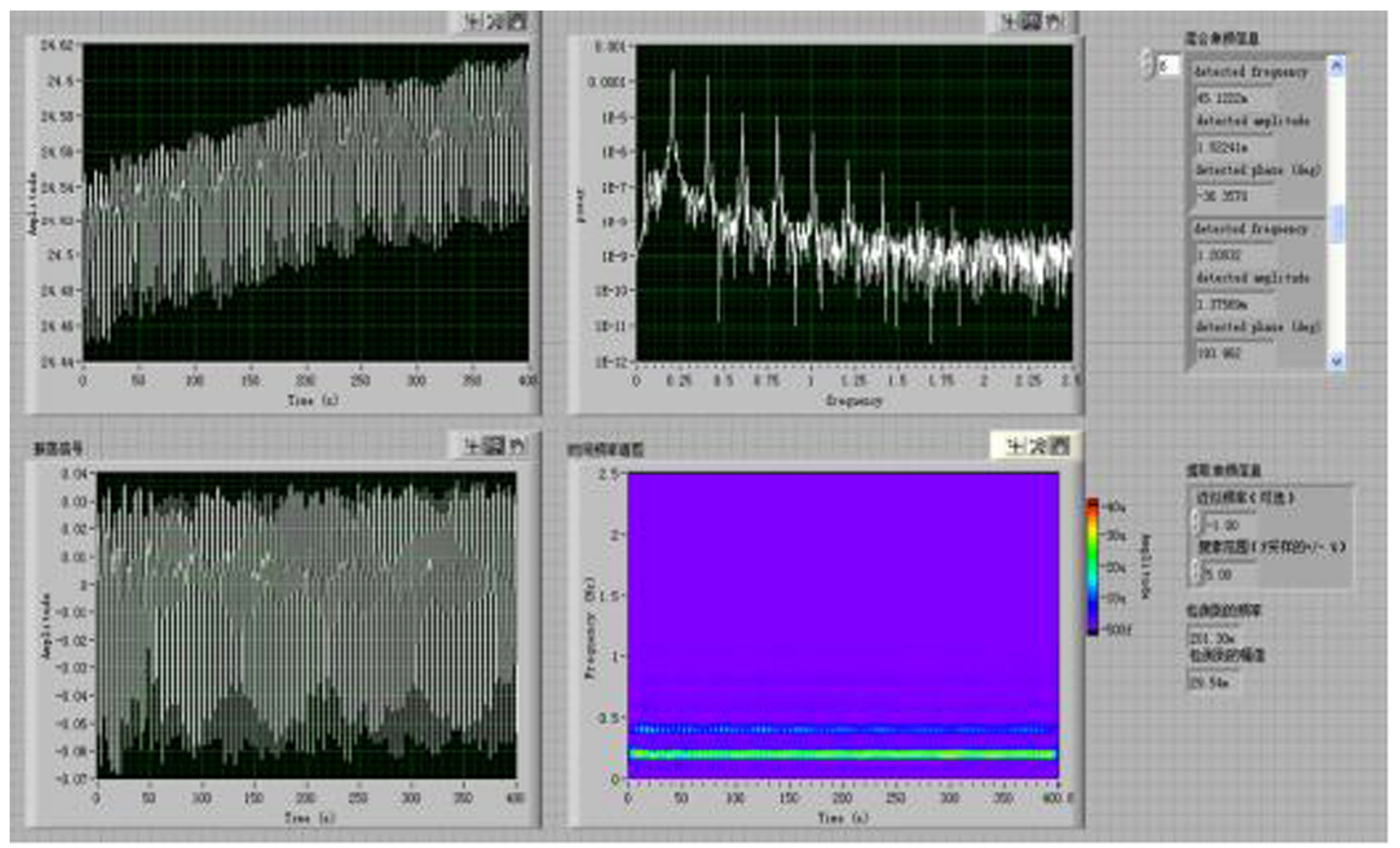

Fig. 3. The sketch map of LabVIEW software platform. 
Table 1

Physical properties of silicone oil.

\begin{tabular}{|c|c|c|c|c|c|c|c|}
\hline Silicone oil (cSt) & $v\left(\mathrm{~m}^{2} \cdot \mathrm{s}^{-1}\right)$ & $\rho\left(\mathrm{kg} \cdot \mathrm{m}^{-3}\right)$ & $\beta\left({ }^{\circ} \mathrm{C}^{-1}\right)$ & $\kappa\left(\mathrm{m}^{2} \cdot \mathrm{s}^{-1}\right)$ & $\sigma\left(\mathrm{N} \cdot \mathrm{m}^{-1}\right)$ & $\partial \sigma / \partial T\left(\mathrm{~N} \cdot \mathrm{m}^{-1} \cdot{ }^{\circ} \mathrm{C}^{-1}\right)$ & $\operatorname{Pr}$ \\
\hline 1 & $1 e-6$ & 818 & $1.29 \mathrm{e}-3$ & $6.19 e-8$ & $1.69 \mathrm{e}-2$ & $-7.55 e-5$ & 16 \\
\hline 1.5 & $1.5 e-6$ & 852 & $1.27 e-3$ & $5.95 e-8$ & $1.77 e-2$ & $-7.35 e-5$ & 25 \\
\hline
\end{tabular}

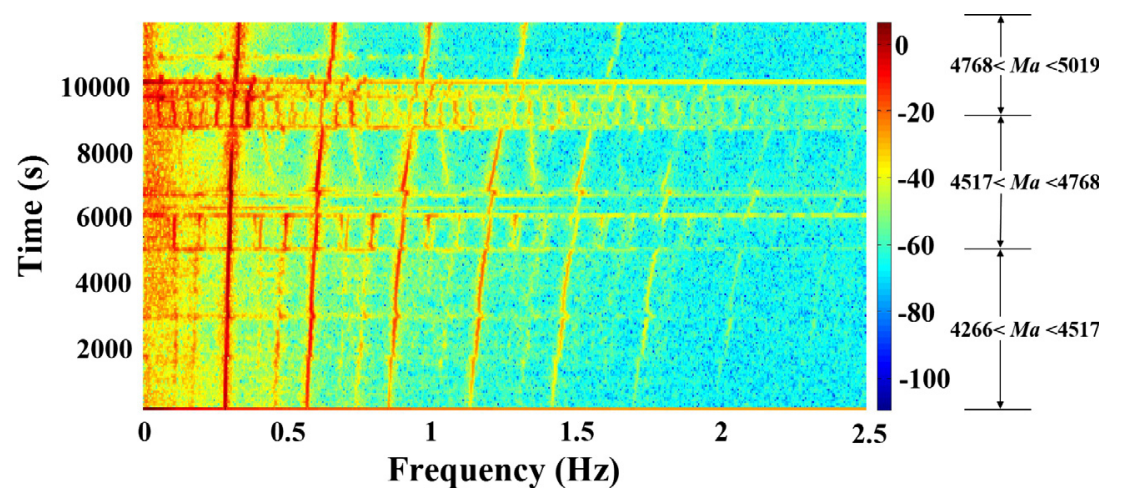

Fig. 4. Time-frequency spectrum of a temperature oscillation series when $\operatorname{Pr}=25$ and $\Gamma=17.3$.

only one frequency in time-frequency spectrum. When the time is approximately $5000 \mathrm{~s}$, three frequencies will appear distinctly. And when time to $9000 \mathrm{~s}$, six frequencies will come into being, which will last for a period. Besides, the fundamental frequency increases over time because the Marangoni number is increasing gradually. And the variation of $M a$ has also been displayed in Fig. 4. In this experimental process, $M a$ range from 4266 to 4517 corresponds to a period of time from 0 to $5000 \mathrm{~s}$. Then $M a$ range from 4517 to 4768 corresponds to time from 5000 to 9000 s. Finally Ma range from 4517 to 4768 corresponds to the period of time beyond $9000 \mathrm{~s}$. Therefore, $M a$ has been selected the control parameter in the following experiment and analysis.

A series of power spectrum analyses on single-point temperature oscillations in liquid layers are performed, shown in Fig. 5. Actually, the determination of the fundamental frequency is of key importance in the power spectrum. The fundamental frequency is the one when the regular oscillation in convection appears for the first time. And then the fundamental frequency changes little once it has been determined in every experimental period. Hence, the fundamental frequency is not always the one with the maximal amplitude of frequency peak, because the power of other frequencies may also be beyond the power of the fundamental frequency with the enhancement of convection. As is shown in Fig. 5(a), when $M a=4266$, thermocapillary flow first destabilizes to oscillate periodically, $f_{1}$ is the fundamental frequency in the power spectrum. When $M a=4517$, shown in Fig. 5 (b), sub-harmonics of one-third and two-thirds of the fundamental frequency ( $1 / 3 f_{1}$ and $2 / 3 f_{1}$ ) will appear in the power spectrum. As the Marangoni number increases to 4768 , sub-harmonics of from $1 / 6 f_{1}$ to $5 / 6 f_{1}$ can be identified, as shown in Fig. $4(\mathrm{c})$. The relationship between the fundamental frequency and other sub-harmonics has been listed in Table 2. However, the tangent bifurcation is instable, and it will degenerate to the periodic oscillation over time. This is the process of tangent bifurcation.

Another transition route is the tangent bifurcation cascade along with the quasi-periodic bifurcation. We can identify the transition route in the experimental condition of $\operatorname{Pr}=16$ and $\Gamma=14.9$ from Fig. 6. The transition process is composed of two different types of bifurcations in a long experimental run. As is shown in power spectra, thermocapillary flow starts oscillating periodically with the fundamental frequency of $f_{1}$ in Fig. 6(a). Then the second fundamental frequency $f_{2}$ appears in Fig. 6(b), and all the other fre-
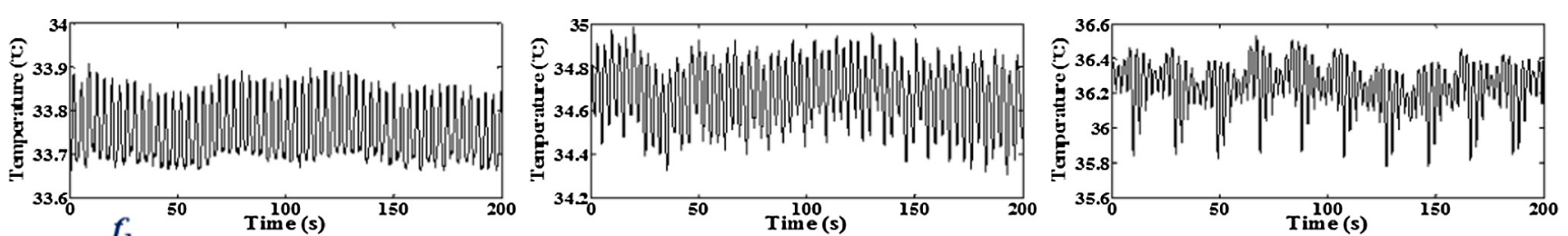

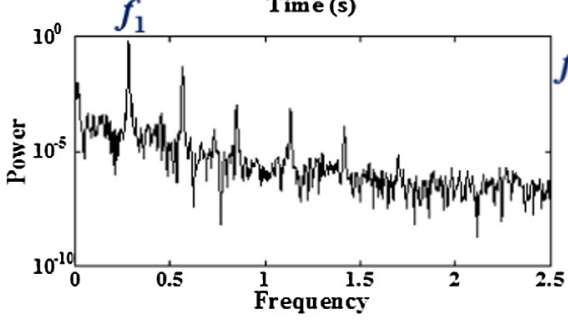

(a)

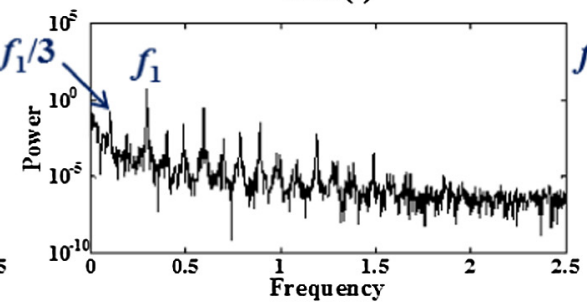

(b)

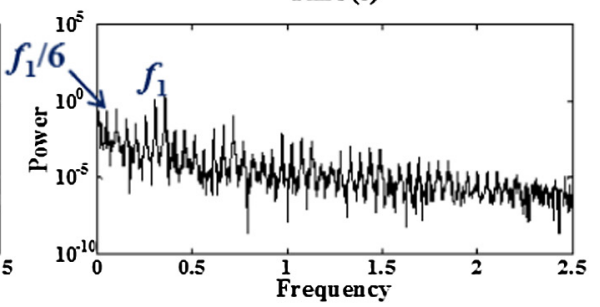

(c)

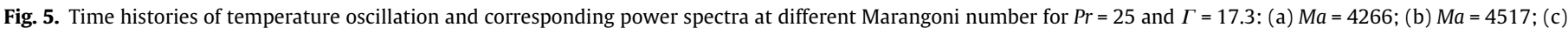
$M a=4768$. 
Table 2

The relationship between the fundamental frequency and other sub-harmonics at different Marangoni number in the experimental condition of $P r=25$ and $\Gamma=17.3$.

\begin{tabular}{|c|c|c|c|}
\hline & The periodic bifurcation & The period-tripling bifurcation & The period-sextupling bifurcation \\
\hline$M a$ & 4266 & 4517 & 4768 \\
\hline The fundamental frequency & $0.290\left(f_{1}\right)$ & $0.298\left(f_{1}\right)$ & $0.308\left(f_{1}\right)$ \\
\hline Other sub-harmonics & $0.580\left(2 f_{1}\right), 0.870\left(3 f_{1}\right), \ldots$ & $\begin{array}{l}0.105\left(1 / 3 f_{1}\right), 0.193\left(2 / 3 f_{1}\right), 0.405\left(4 / 3 f_{1}\right) \\
0.490\left(5 / 3 f_{1}\right), 0.598\left(2 f_{1}\right), \ldots\end{array}$ & $\begin{array}{l}0.048\left(1 / 6 f_{1}\right), 0.103\left(2 / 6 f_{1}\right), 0.160\left(3 / 6 f_{1}\right) \text {, } \\
0.205\left(4 / 6 f_{1}\right), 0.255\left(5 / 6 f_{1}\right), \ldots\end{array}$ \\
\hline
\end{tabular}
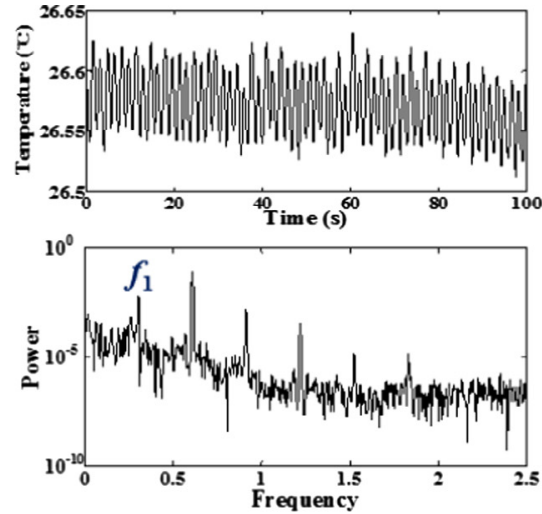

(a)
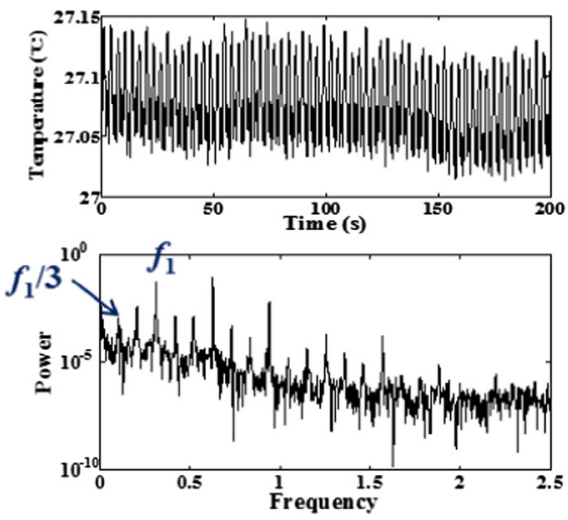

(d)
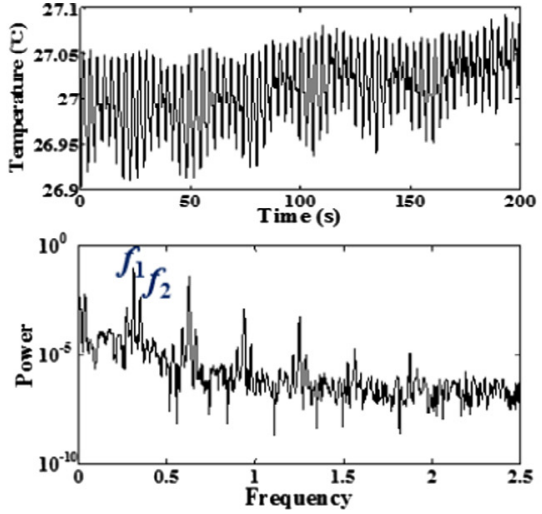

(b)
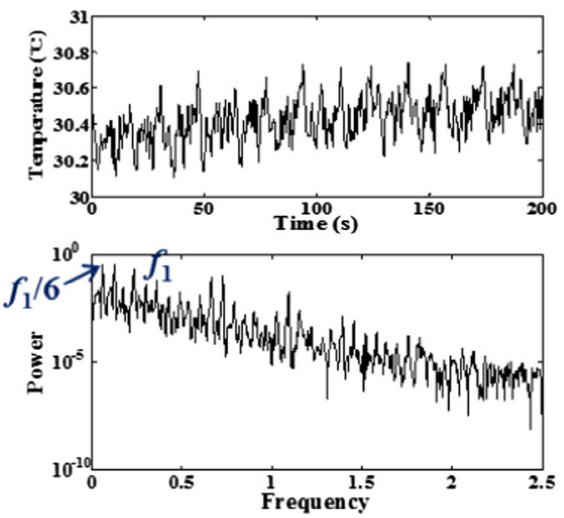

(e)
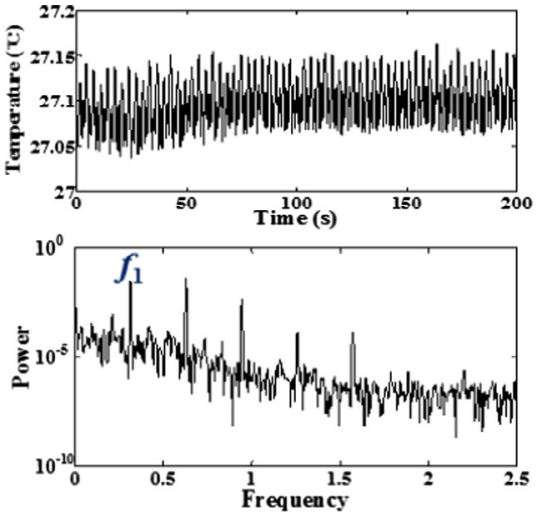

(c)
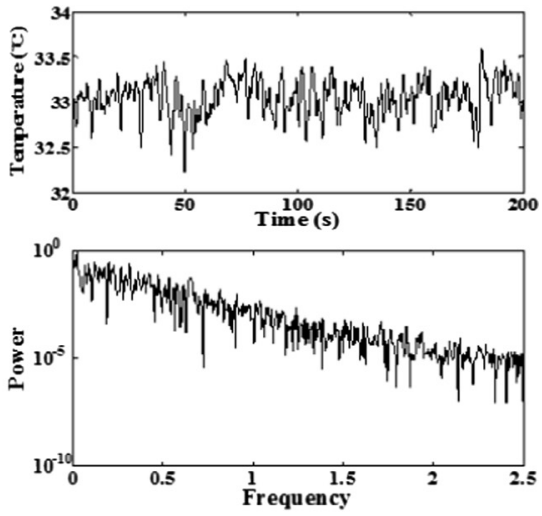

(f)

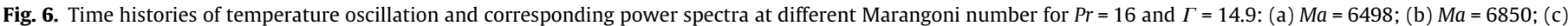
$M a=7025$; (d) $M a=7728$; (e) $M a=9133$; (f) $M a=10,538$.

quencies can be expressed by the linear combination of the two fundamental frequencies $f_{1}$ and $f_{2}$. This bifurcation is known as the quasi-periodic bifurcation, which is the similar experimental finding of Zhu et al. [10]. With the increase of Marangoni number, thermocapillary convection reverse to periodic motion in Fig. 6(c). Following a further increase in the Marangoni number, a periodtripling bifurcation and a period-sextupling bifurcation will appear in order in Fig. 6(d) and (e). Finally, the convection transits to aperiodic motion in Fig. 6(f).

In addition, there is another type tangent bifurcation accompanied with the quasi-periodic bifurcation. As is shown in Fig. 7, a bifurcation cascade can be acquired in the experimental condition of $\operatorname{Pr}=25$ and $\Gamma=20.8$. When $M a=3601$, thermocapillary convection presents a state of periodic oscillation in Fig. 7(a). When $M a=4008$, the quasi-periodic bifurcation of two frequencies will appear in the convection in Fig. 7(b). When $M a=4415$, shown in Fig. 7(c), we can acquire the period-quintupling bifurcation, which is different from the period-tripling bifurcation.

Actually, the tangent bifurcation is associated with the intermittency [12,13]. In the experimental run, we also observed the transient intermittency while $\operatorname{Pr}=25$ and $\Gamma=20.8$. Periodic motion will be interrupted by short irregular bursts. As is shown in Fig. 8, two points in liquid at different distance away from cold-side and hot-side were chosen to measure temperature oscillations. The occurrence of intermittency in the two measured-points are basically consistent on the time scale, but there are some differences in amplitude. The time interval of intermittency will increase in the experimental period, shown in Fig. 9. In the present experiment, it is difficult to observe the intermittency for a certain experimental condition, so we only focused on the tangent bifurcation transition process in most experiments.

In addition, the tangent bifurcation is an unstable type of bifurcations, and it has a certain randomness for the appearance of tangent bifurcation in each experiment. Therefore, this bifurcation may appear in various parameters $(\operatorname{Pr}$ and $\Gamma)$.

\subsection{Chaotic characteristic analyses of bifurcations}

The method of phase-space reconstruction [14,15] is welldeveloped method of chaotic structure research, and it could be applied to analyze the chaotic characteristic of tangent bifurcations. Takens [16] has proved the state of many dynamic systems 

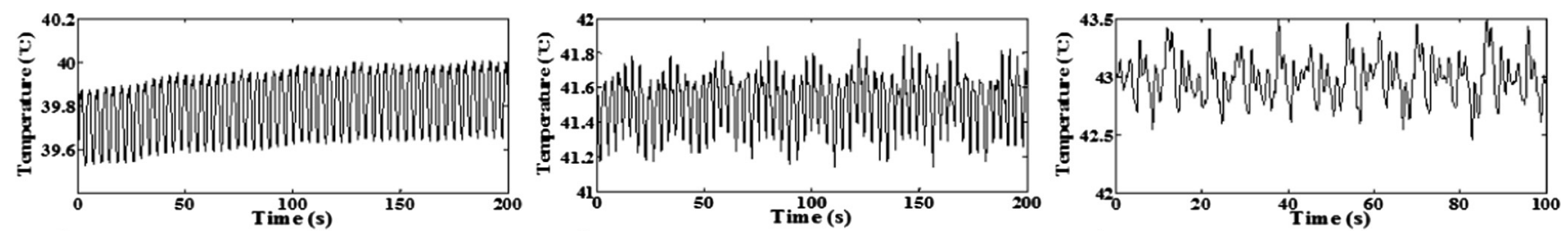

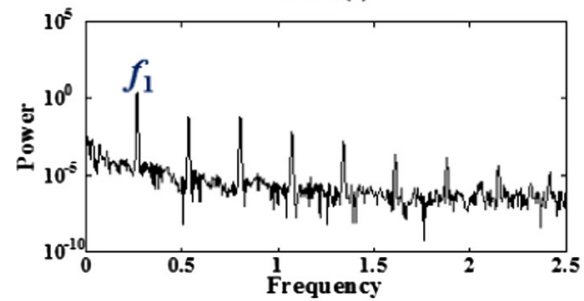

(a)

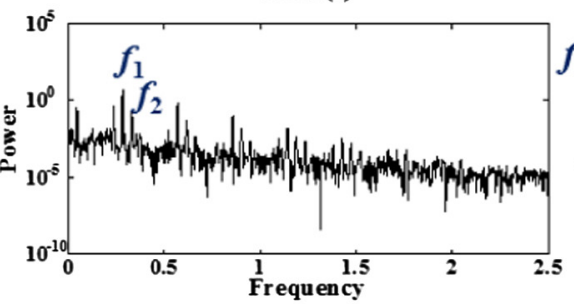

(b)

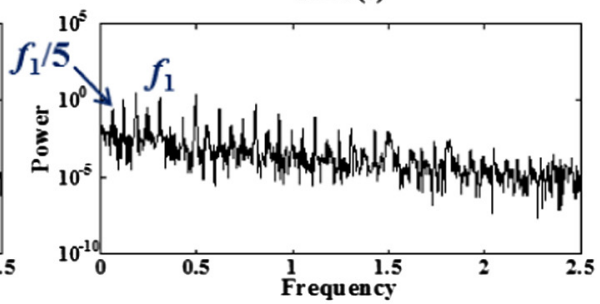

(c)

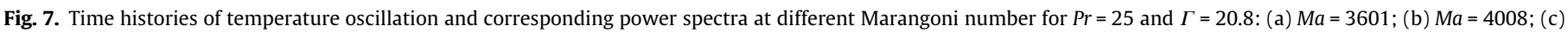
$M a=4415$.

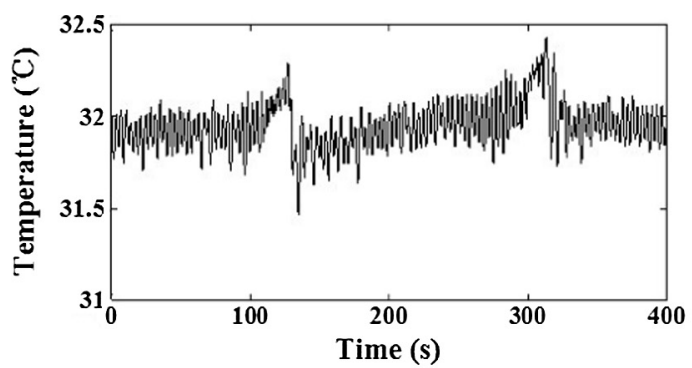

(a)

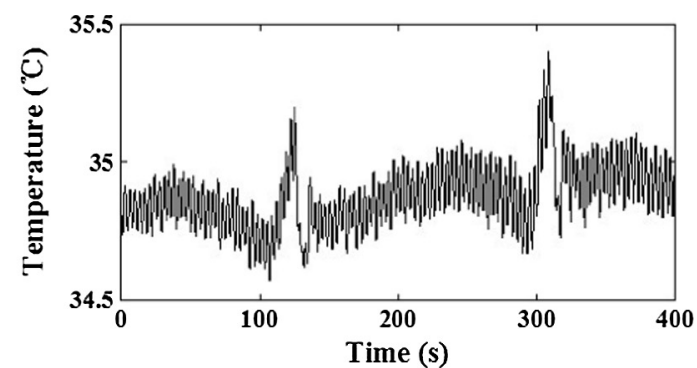

(b)

Fig. 8. Time histories of temperature oscillation at $M a=2928$ when $\mathrm{Pr}=25$ and $\Gamma=20.8$ : (a) measured-pointI; (b) measured-pointII.

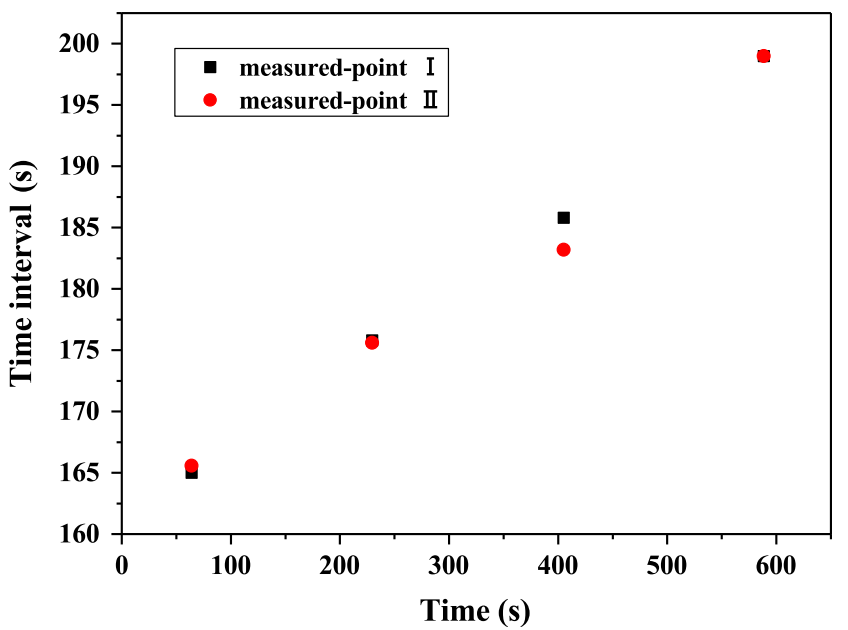

Fig. 9. The time interval of intermittency in an experimental period at $M a=2928$ when $\operatorname{Pr}=25$ and $\Gamma=20.8$.

can be accurately reconstructed by a finite window of the time series. This window is called a time delay. Consider a temperature time series $\{T(t)\}$, and denote the phase-space vectors $v(t)$ :

$v(t)=\{T(t), T(t+\tau), \ldots, T(t+(m-1) \tau)\}$,

where $\tau$ is the time delay, $m$ is the embedding dimension. We can achieve the phase-space reconstruction by selecting appropriate parameters of $\tau$ and $m$.
For one-dimensional temperature data in our experiment, a three-dimensional phase-space based on the time-delay has been reconstructed, and then we could analyze successively the chaotic characteristic in the different bifurcation phase. It is shown in Fig. 10 that the trajectory of temperature oscillation series in the reconstructed phase-space where the axes correspond to $T(t)$, $T(t+\tau)$ and $T(t+2 \tau)$. The embedding dimension is $m=3$, and the time delay could be $\tau=3$. Then one-dimensional time series data can be extended to a three-dimensional space. The experimental data at $P r=25$ and $\Gamma=17.3$ (Fig. 5) could be chosen to analyze the chaotic characteristic of bifurcations in the reconstructed phase-space. The periodic motion is a limit cycle in the phasespace in Fig. 10(a). Three trajectories appear in the motion of period-tripling bifurcation in Fig. 10(b). And when the convection transitions to the phase of period-sextupling bifurcation, there appear six distinct trajectories in the phase-space in Fig. 10(c).

Furthermore, the Lyapunov exponent of a dynamical system is introduced to characterize the rate of separation of infinitesimally close trajectories $[17,18]$.

For a continuous dynamical system in a n-dimensional phase space, we monitor the long-term evolution of an infinitesimal nsphere of initial conditions; the sphere will become a n-ellipsoid due to the locally deforming nature of the flow. The ith onedimensional Lyapunov exponent is then defined in terms of the length of the ellipsoidal principal axis $p_{i}(t)$ :

$\lambda_{i}=\lim _{t \rightarrow \infty} \frac{1}{t} \log _{2} \frac{p_{i}(t)}{p_{i}(0)} \quad(i=1,2, \ldots)$,

where the $\lambda_{i}$ are ordered from largest to smallest: $\lambda_{1} \geq \lambda_{2} \geq \cdots \geq \lambda_{n}$. The largest Lyapunov exponent $\lambda_{1}$ are related to the expanding nat- 


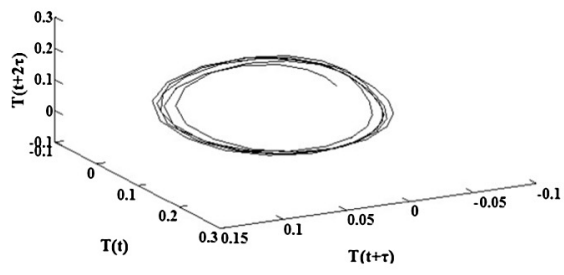

(a)

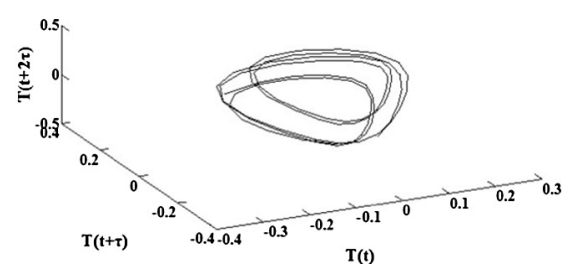

(b)

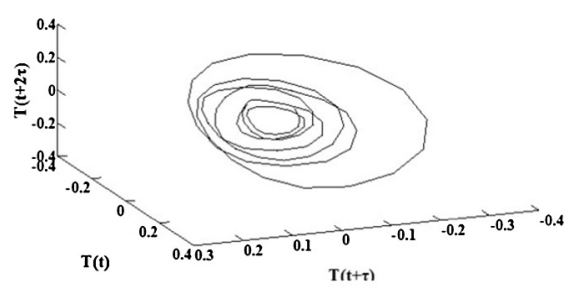

(c)

Fig. 10. Three-dimensional time-delay representation of the temperature for $\operatorname{Pr}=25$ and $\Gamma=17.3$ : (a) $M a=4266$; (b) $M a=4517$; (c) $M a=4768$.

ure of different directions in phase space. When $\lambda_{1}>0$, it signify that close trajectories separate and flow begins to transit to chaos. Wolf method [18] was chosen to calculate the maximum Lyapunov exponent on temperature data in the phase-space. Through this method, the largest Lyapunov exponents in periodic, period-tripling and period-sextupling bifurcation phases, as shown in Table 3, could be calculated successively. They are in turn: $0.079,0.193$ and 0.227. The maximum Lyapunov exponent increases with the Marangoni number increasing, and phase trajectories separate at a faster rate.

By the same token, the permutation entropy method $[19,20]$ is introduced to evaluate the chaotic characteristic of bifurcations. Reconsider the temperature time series $\left\{T\left(t_{i}\right), i=1,2, \ldots, n\right\}$ and the phase-space vectors $v\left(t_{i}\right)$ :

$v\left(t_{i}\right)=\left(T\left(t_{i}\right), T\left(t_{i}+\tau\right), \ldots, T\left(t_{i}+(m-1) \tau\right)\right), t_{i}(i=1,2, \ldots, n)$

where $\tau$ is the time delay, $m$ is the embedding dimension, and $n$ is the number of data. For the sequence, we indexed all the possible $m$ ! permutations of order $m \geq 2$ defined as $\pi$. For all the vectors $v$ $\left(t_{i}\right)$, we count the number of permutations, denoted by $q(\pi)$, and then calculate the relative frequency $p(\pi)$ :

$p(\pi)=\frac{q(\pi)}{n-(m-1) \tau f}$,

where $f$ is the sampling rate. The permutation entropy $H(m)$ is defined as

$H(m)=-\sum_{\pi} p(\pi) \log _{2} p(\pi)$

Then it can be normalized as follow:

$h_{p}=\frac{H(m)}{\log _{2} m !}$.

Therefore, $h_{p}$ is in the range of $0-1$. This is the finally permutation entropy of the temperature time series. The size of $h_{p}$ indicates the degree of randomness of the time series. The lower $h_{p}$ equates to more regular time series; whereas the higher $h_{p}$ signifies that the time series is more close to stochastic state. The permutation entropy of three types of bifurcations in Fig. 10 can also be calculated. The $h_{p}$ in Table 3 is in turn: $0.578,0.587$ and 0.668 , which also signifies the time series become more and more irregular.

Table 3

The permutation entropy $h_{p}$ in different Marangoni number.

\begin{tabular}{lll}
\hline$M a$ & $\lambda_{1}$ & $h_{p}$ \\
\hline 4266 & 0.079 & 0.578 \\
4517 & 0.193 & 0.587 \\
4768 & 0.227 & 0.668 \\
\hline
\end{tabular}

\section{Conclusion}

In the present paper, transition routes of tangent bifurcations in thermocapillary convection are investigated in particular. There are different types of tangent bifurcations, including periodtripling bifurcation, period-quintupling bifurcation and the period-tripling bifurcation along with the quasi-periodic bifurcation, et al. In addition, transient intermittency could be observed in an experimental run.

The phase-space reconstruction is introduced to qualitatively analyze the chaotic characteristic of tangent bifurcations, and trajectories differ in different bifurcation phases. Moreover, analytical methods of maximum Lyapunov exponent and permutation entropy are applied to quantificationally analyze the chaotic characteristic of bifurcations to a certain extent.

\section{Acknowledgment}

This work was supported by SJ-10 Satellite Program of the Strategic Pioneer Program on Space Science, Chinese Academy of Sciences, under grant No. XDA04020405, XDA04020202-05, the China Manned Space Engineering program (TG-2), Cooperative Research Project between China and Russia, and the Natural Science Foundation Project (11372328).

\section{References}

[1] Li Duan, Qi Kang, Hu Wenrui, Characters of surface deformation and surface wave in thermal capillary convection, Sci. China Ser. E: Technol. Sci. 49 (5) (2006) 601-610.

[2] M. Hamed, J.M. Floryan, Marangoni convection. Part 1. A cavity with differentially heated sidewalls, J. Fluid Mech. 405 (2000) 79-110.

[3] J.P. Gollub, S.V. Benson, Many routes to turbulent convection, J. Fluid Mech. 100 (3) (1980) 449-470.

[4] D. Mukutmoni, Thermal convection in small enclosures: an atypical bifurcation sequence, Int. J. Heat Mass Transf. 38 (1) (1995) 113-126.

[5] E. Bucchignani, S. Fulvio, Rayleigh-Bénard convection in limited domains: Part 1 - Oscillatory flow, Numer. Heat Transf. Part A: Appl.: Int. J. Comput. Methodol. 36 (1) (1999) 1-16.

[6] E. Bucchignani, S. Fulvio, Rayleigh-Bénard convection in limited domains: Part 2 - Transition to chaos, Numer. Heat Transf. Part A: Appl.: Int. J. Comput. Methodol. 36 (1) (1999) 17-34.

[7] Yok-Sheung Li, Zhi-Wu Chen, Jie-Min Zhan, Double-diffusive Marangoni convection in a rectangular cavity: transition to chaos, Int. J. Heat Mass Transf. 53 (23-24) (2010) 5223-5231.

[8] Jia-Jia Yu, Chun-Mei Wu, You-Rong Li, Jie-Chao Chen, Thermal-solutal capillary-buoyancy flow of a low Prandtl number binary mixture with the capillary ratio of -1 in an annular pool, Phys. Fluids 28 (2016) 084102.

[9] Wenrui Hu, Zemei Tang, Kai Li, Floating zone thermocapillary convection, Adv. Mech. 39 (3) (2009) 360-377.

[10] Peng Zhu, Li Duan, Qi Kang, Transition to chaos in thermocapillary convection, Int. J. Heat Mass Transf. 57 (2) (2013) 457-464.

[11] Peng Zhu, Li Duan, Qi Kang, Characteristics of surface oscillation in thermocapillary convection, Exp. Therm. Fluid Sci. 35 (2011) 1444-1450.

[12] Edward Ott, Chaos in Dynamical Systems, second ed., Cambridge University Press, Oxford, 2002.

[13] J.C. Sprott, Chaos and Time-Series Analysis, Cambridge University Press, Oxford, 2003.

[14] Bin Chen, The Correlation of Chaotic Waveform: Phase Space Trajectory and Autocorrelation Characteristics of Chaotic Sequences, Xidian University Press, Xi'an, 2011. 
[15] Yan Aa, Kai Li, Zemei Tang, Zhonghua Cao, Wenrui Hu, Period-doubling bifurcations of the thermocapillary convection in a floating half zone, Sci. China Tech. Sci. 53 (9) (2010) 1681-1686.

[16] F. Takens, Detecting strange attractors in turbulence, in: D. Rand, L.S. Young (Eds.), Dynamical Systems and Turbulence, Warwick 1980, Lecture Notes in Mathematics, vol. 898, Springer-Verlag, Berlin Heidelberg, 1981, pp. 366-381.

[17] G. Benettin, L. Galgani, A. Giorgilli, J.-M. Strelcyn, Lyapunov characteristic exponents for smooth dynamical systems and for Hamiltonian systems; method for computing all of them, Meccanica 15 (1) (1980) 9-20.
[18] Alan Wolf, Jack B. Swift, Harry L. Swinney, John A. Vastano, Determining Lyapunov exponents from a time-series, Physica D 16 (3) (1985) 285-317.

[19] Christoph Bandt, Bernd Pompe, Permutation entropy: a natural complexity measure for time series, Phys. Rev. Lett. 88 (17) (2002) 174102.

[20] T. Matsugase et al., Transition to chaotic thermocapillary convection in a half zone liquid bridge, Int. J. Heat Mass Transf. 89 (2015) 903-912. 